\title{
Calcifilaxis. Comunicación de un caso
}

\author{
Calciphylaxis. Case report
}

\author{
Fabiola Jiménez-Hernández, ${ }^{*}$ Luisa Fernanda Baldassarri-Ortego, ${ }^{\ddagger}$ Valeria Álvarez-Rivero§
}

\section{RESUMEN}

La calcifilaxis se define como la isquemia o necrosis cutánea de dermis y tejido celular subcutáneo que se manifiesta clínicamente por la presencia de úlceras crónicas y dolorosas ocasionadas por calcificación vascular, la cual se desarrolla principalmente en pacientes en terapia con diálisis peritoneal por insuficiencia renal crónica. Una vez realizado el diagnóstico, el pronóstico es bastante desalentador. En este artículo comunicamos el caso y la evolución de una paciente de 58 años con calcifilaxis.

Palabras clave: Calcifilaxis, úlceras crónicas, enfermedad renal crónica, diálisis peritoneal.

\section{ABSTRACT}

Calciphylaxis is a condition caused by the ischemia and necrosis of dermis and subcutaneous tissue in patients undergoing dialysis due to chronic kidney disease. It is clinically characterized by chronic, painful-non healing ulcers. The prognosis is rather bleak. In this article, we present the case and evolution of a 58 year old, female patient with the diagnosis of calciphylaxis secondary to chronic kidney disease.

Keywords: Calciphylaxis, chronic ulcers, chronic kidney disease, peritoneal dialysis.

\section{INTRODUCCIÓN}

La calcifilaxis fue descrita por primera vez por Hans Seyle en $1961{ }^{1}$

Conocida también como arteriolopatía calcificante urémica, se le define como la necrosis o isquemia cutánea por calcificación vascular, lo cual provoca la oclusión de la vasculatura dérmica y del tejido celular subcutáneo, ocasionando a nivel cutáneo el desarrollo de úlceras crónicas y dolorosas. ${ }^{1}$

\section{Epidemiología}

La calcifilaxis puede presentarse en $1-4 \%^{2,3}$ de los pacientes en tratamiento con diálisis peritoneal crónica, aproximadamente 2.5 años después de haber iniciado el procedimiento. ${ }^{1,4}$

\footnotetext{
* Dermatóloga.

‡ Residente del tercer año de Dermatología.

$\S$ Residente del primer año de Dermatología.
}

Centro Dermatológico «Dr. Ladislao de la Pascua», SSCDMX.

\section{Fisiopatogenia}

Las alteraciones metabólicas observadas en los pacientes renales crónicos en terapia de sustitución renal, como la hiperfosfatemia e hipocalcemia, provocan un desajuste en el balance entre los promotores e inhibidores de calcificación vascular, ${ }^{5}$ favoreciendo el cambio de las células de músculo liso a un fenotipo osteoblástico, lo cual promueve la generación de depósitos de cristales de hidroxiapatita de calcio en la capa media de los vasos sanguíneos. ${ }^{6}$ Por otro lado, el uso de antagonistas de la vitamina $\mathrm{K}$ como la warfarina, que es un medicamento comúnmente empleado en este grupo de pacientes, inhibe la producción de la proteína de matriz extracelular Gla, inhibidor potente de calcificación de los vasos sanguíneos. ${ }^{5}$

Los depósitos de los cristales de calcio y la trombosis vascular de los vasos sanguíneos en las paredes de la microvasculatura se extienden hasta afectar los vasos arteriales más grandes, ocasionando necrosis cutánea.

Citar como: Jiménez-Hernández F, Baldassarri-Ortego LF, Álvarez-Rivero V. Calcifilaxis. Comunicación de un caso. Rev Cent Dermatol Pascua. 2020; 29 (2-3): 77-80. https://dx.doi.org/10.35366/99919 


\section{Cuadro clínico}

Habitualmente las lesiones se ubican en el tercio proximal de extremidades inferiores así como en la región abdominal, sitios donde el tejido adiposo suele ser más abundante.$^{7,8}$ Es bilateral y con tendencia a la simetría. Inicia con áreas dolorosas, bien circunscritas, de color violáceo, en las que posteriormente se desarrolla necrosis, dando lugar a úlceras crónicas, muy dolorosas, de bordes cortados a pico, refractarias a tratamiento. Frecuentemente se superinfectan, la piel circundante se palpa infiltrada, indurada. Puede acompañarse de livedo reticularis. ${ }^{9}$

\section{Diagnóstico}

El diagnóstico se basa en las características clínicas, y por los antecedentes personales patológicos del paciente. Para corroborar el diagnóstico se puede realizar una biopsia de piel; sin embargo, las complicaciones del procedimiento pueden llegar a ser mayores que los beneficios. ${ }^{10}$ En el estudio histopatológico de la pieza destaca la presencia de calcificación de la capa media, fibrosis de la íntima y trombosis vascular en vasos sanguíneos de dermis profunda y tejido celular subcutáneo. La tinción con Von Kossa demuestra la presencia de calcificación en los vasos sanguíneos. ${ }^{9}$

La tomografía computarizada SPECT-CT muestra los sitios de calcificación vascular. Tiene sensibilidad de $87 \%$ y especificidad de $97 \% .^{11,12}$

Los diagnósticos diferenciales incluyen úlceras por insuficiencia venosa o arterial y por neuropatía diabética, así como úlcera hipertensiva de Martorell, pioderma gangrenoso y émbolos de colesterol. Es importante identificar la causa, ya que el tratamiento y pronóstico de cada una de estas entidades es distinto. ${ }^{7}$

\section{Tratamiento}

El tratamiento debe ser multidisciplinario, en conjunto con el médico dermatólogo, nefrólogo, cirujano plástico y personal de cuidados paliativos. ${ }^{5}$ Debe estar encaminado a cuatro puntos principales:

1. Detener la calcificación vascular. Se sugiere la realización de hemodiálisis cinco veces por semana para mejorar el estado metabólico del paciente.

2. Descalcificación de vasos calcificados. El tiosulfato de sodio es un quelante de sodio que hace al calcio más soluble en agua para favorecer su eliminación a través de la diálisis. Puede administrarse a dosis de 5-25 mg IV al terminar, o después de la diálisis, y debe mantenerse dos meses después de la remisión del cuadro.

3. Manejo de las heridas. No se recomienda desbridación quirúrgica debido a que el daño arterial existente complicaría la evolución. En caso de lesiones infectadas y con exudado puede realizarse tratamiento avanzado de heridas, aplicando apósitos antibacterianos o terapia dirigida, previo cultivo del agente patógeno. Los tratamientos con dispositivos de presión negativa y con oxígeno hiperbárico pueden ser de utilidad para el tratamiento de úlceras refractarias al manejo estándar. ${ }^{13}$

4. Manejo del dolor. Para la analgesia se prefiere el uso de fentanilo y metadona en pacientes con daño renal. ${ }^{7,14}$

\section{Pronóstico}

Una vez corroborado el diagnóstico, el pronóstico es bastante desalentador, con probabilidad de supervivencia a un año en $50 \%$ de los casos, y a dos años en el $20 \% .{ }^{4}$ Las patologías asociadas como la anemia o la proteinuria dificultan los tratamientos para favorecer la cicatrización, por lo que el manejo se limita a controlar la infección y el dolor. La mortalidad asciende hasta $60 \%$ en caso de afectar tronco, miembros superiores y pene. ${ }^{7}$

\section{CASO CLÍNICO}

Paciente del sexo femenino, de 58 años, residente de la Ciudad de México, con insuficiencia renal crónica estadio V secundaria a hipertensión arterial sistémica, diagnosticada dos años antes, en tratamiento por servicio médico institucional con losartán, atorvastatina y eritropoyetina. Desde hace dos meses se encuentra en terapia de sustitución de la función renal con hemodiálisis tres veces a la semana. Acude a la consulta externa de dermatología por presentar desde hace 18 meses, una dermatosis diseminada a miembros inferiores que afecta cara externa de piernas derecha e izquierda, en tercio distal, bilateral y con tendencia a la simetría, constituida por dos úlceras, la mayor de ellas de aproximadamente de $6 \times 4 \mathrm{~cm}$, de forma irregular, con tejido de granulación y necrosis en la base, con bordes en sacabocado, regulares y bien definidos, con olor fétido, eritema, induración y costras en la periferia. De evolución crónica y muy dolorosa (Figuras 1 y 2). No se palpan los pulsos pedio y tibial posteriores de forma bilateral, y el estudio con ultrasonido Doppler manual demostró disminución del flujo vascular en 
ambas piernas. Se realiza una angiorresonancia que demuestra la presencia de calcificación arterial de miembros inferiores con insuficiencia arterial (Figura 3). Se solicitó la realización de un cultivo de la secreción de una de las úlceras, donde se observó desarrollo de Morganella morganii, sensible a amikacina, cefepima, ciprofloxacino y cefoxitina.

El estudio histopatológico se difiere debido a la insuficiencia arterial bilateral con la que cursa la paciente, lo cual contraindica su realización. Se establece el diagnóstico definitivo de úlceras distales por calcifilaxis secundaria a enfermedad renal crónica, y se inicia tratamiento con un esquema antibiótico con ciprofloxacino $500 \mathrm{mg}$ cada ocho horas por 14 días, fomentos secantes, analgésicos opioides, desbridantes enzimáticos en crema y cicatrizantes tópicos. Se interconsulta a los servicios de nefrología para ajustar manejo de hemodiálisis, así como angiología para mejorar la función arterial.

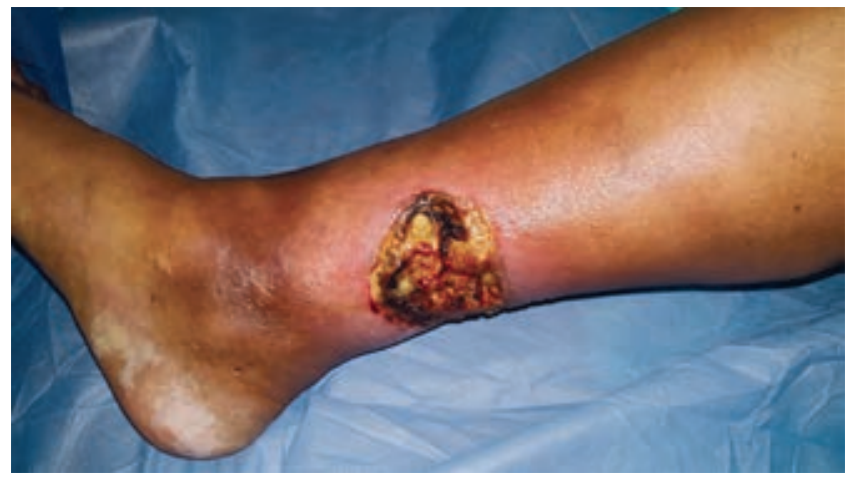

Figura 1: Úlcera en tercio distal de cara interna de extremidad inferior izquierda.

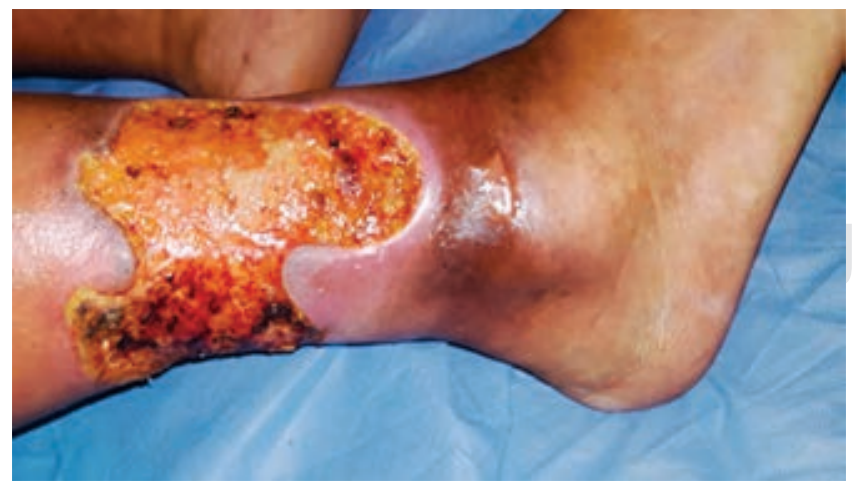

Figura 2: Úlcera en tercio distal de cara externa de extremidad inferior derecha.
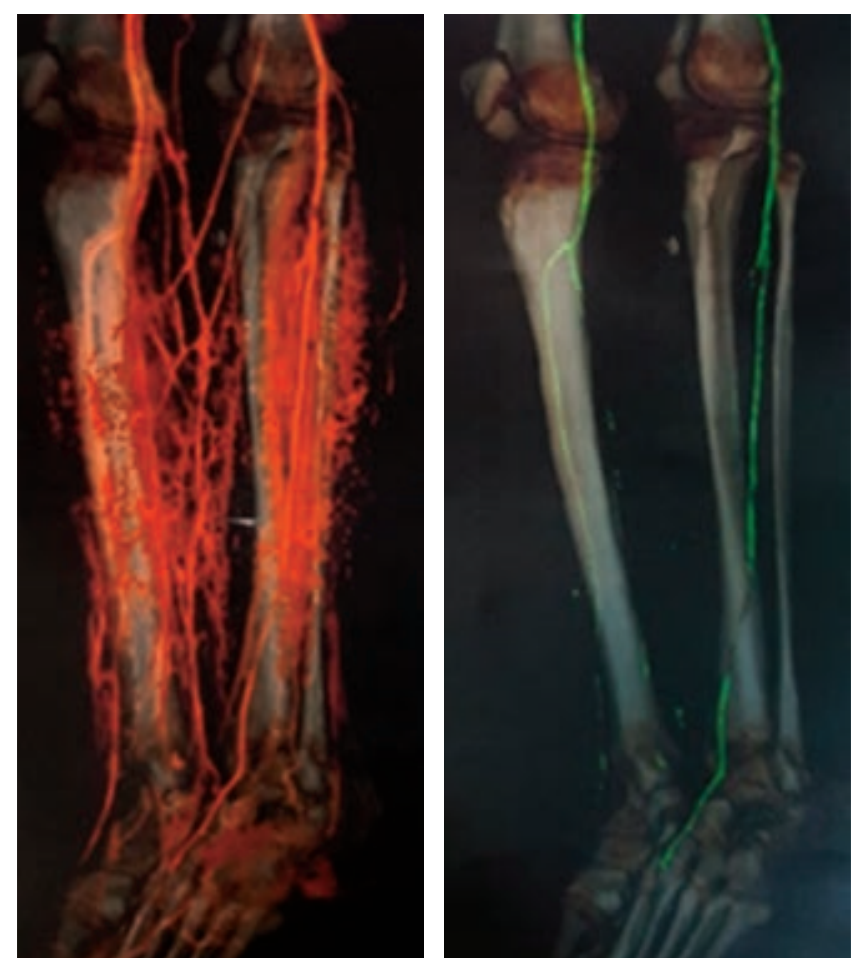

Figura 3: Angiorresonancia magnética se destaca la ausencia de flujo vascular en ambas piernas.

Desafortunadamente, la paciente falleció poco tiempo después de acudir a este centro por complicaciones de la insuficiencia renal crónica con que cursaba.

\section{CONCLUSIÓN}

La calcifilaxis es un padecimiento que se observa en pacientes con enfermedad renal crónica en tratamiento de sustitución renal, manifestándose como úlceras dolorosas, crónicas, refractarias a tratamiento. El diagnóstico suele ser clínico y se recomienda un manejo multidisciplinario para prevención de nuevos depósitos de calcio en los vasos sanguíneos, remoción del calcio ya existente y manejo adecuado de las heridas y del dolor del paciente. El pronóstico es desalentador, con una mortalidad de $50 \%$ de los pacientes que presentan úlceras al año posterior a la aparición de las lesiones.

\section{REFERENCIAS}

1. Saito T, Mima Y, Sugiyama M, Miyazawa N, lida A, Kanazawa N et al. Multidisciplinary management of calciphylaxis: a series of 5 patients at a single facility. CEN Case Rep. 2019; (0123456789). Available in: https://doi.org/10.1007/s13730-019-00439-8. 
2. Nigwekar SU, Wolf M, Sterns RH, Hix JK. Calciphylaxis from nonuremic causes: a systematic review. Clin J Am Soc Nephrol. 2008; 3: 1139-1143.

3. Nigwekar SU. Calciphylaxis. Curr Opin Nephrol Hypertens. 2017; 26: 276-281.

4. Gaisne R, Péré M, Menoyo V, Hourmant M, Larmet-Burgeot D. Calciphylaxis epidemiology, risk factors, treatment and survival among French chronic kidney disease patients: a case-control study. BMC Nephrol. 2020; 21: 1-11.

5. Seethapathy H, Noureddine L. Calciphylaxis: approach to diagnosis and management. Adv Chronic Kidney Dis. 2019; 26: 484-490. Available in: https://doi.org/10.1053/j.ackd.2019.09.005.

6. Nigwekar SU, Thadhani R, Brandenburg VM. Calciphylaxis. N Engl J Med. 2018; 378: 1704-1714.

7. García-Lozano JA, Ocampo-Candiani J, Martínez-Cabriales SA, Garza-Rodríguez V. An update on calciphylaxis. Am J Clin Dermatol. 2018; 19: 599-608.

8. Meegada S, Annakula M, Challa T, Peddi P. Calciphylaxis: a deceiving cellulitis. Cureus. 2019; 11: e6518.

9. Colboc H, Moguelet P, Bazin D, Carvalho P, Dillies AS, Chaby G et al. Localization, morphologic features, and chemical composition of calciphylaxis-related skin deposits in patients with calcific uremic arteriolopathy. JAMA Dermatology. 2019; 155: 789-796.

10. Musso CG, Enz PA, Kowalczuk A, Cozzolino M, Brandenburg V, Nigwekar S. Differential diagnosis of calciphylaxis in chronic dialysis patients. Int Urol Nephrol. 2020; 52: 595-597. Available in: https://doi. org/10.1007/s11255-020-02388-z.

11. Faizi N, Kazmi S. Universal health coverage. There is more to it than meets the eye. J Fam Med Prim Care. 2017; 6: 169-170. Available in: http://www.jfmpc.com/article.asp?issn=2249-4863;year=2017;volum e=6;issue=1; spage=169; epage=170; aulast=Faizi.

12. Herrera-Martínez Y, Martín-Marcuartu JJ, Mohigefer-Barrera J, Jiménez-Hoyuela JM, García-Jiménez R. Imagen híbrida (SPECT/ TC) en el diagnóstico precoz de un paciente con calcifilaxis. Nefrología. 2020; 40: 576-578. Available in: https://doi.org/10.1016/j. nefro.2019.11.003.

13. Dado DN, Huang B, Foster DV, Nielsen JS, Gurney JM, Morrow BD et al. Management of calciphylaxis in a burn center: a case series and review of the literature. Burns. 2019; 45: 241-246. Available in: https://doi.org/10.1016/j.burns.2018.09.008.

14. Chang JJ. Calciphylaxis: diagnosis, pathogenesis and treatment. Adv Skin Wound Care. 2019; 32: 205-215.

\section{Correspondencia:}

Dra. Fabiola Jiménez Hernández Dr. Vértiz Núm. 464 Esq. Eje 3 Sur, Col. Buenos Aires, 06780, Alcaldía Cuauhtémoc, CDMX Tels. 55 5538-7033 y 55 5519-6351 E-mail: drajimenezher@gmail.com 